\title{
Differences in Structural and Electronic Properties of Conducting LGO/STO and LAO/STO Interfaces
}

\author{
C. Cantoni*, J. Gazquez*, M. P. Oxley*, A. R. Lupini*, M. Varela*, S. J. Pennycook*, P. Perna ${ }^{\dagger}$, D. \\ Marre $^{\S}$, C. Aruta ${ }^{\dagger}$, U. Scotti di Uccio ${ }^{\dagger}$, F. Miletto Granozio ${ }^{\dagger}$. \\ *Materials Science and Technology Division, Oak Ridge National Laboratory, TN 37831, USA \\ ${ }^{\dagger}$ Dipartimento di Scienze Fisiche, CNR-SPIN Universita` di Napoli “Federico II”, 80126 Naples, \\ Italy \\ ${ }^{\S}$ Dipartimento di Fisica, CNR-SPIN, Universita' di Genova, via Dodecaneso 33, 16146 Genova, \\ Italy
}

The formation of a 2-dimensional electron liquid with tunable conductivity and superconductivity at the interface between band insulators $\mathrm{SrTiO}_{3}$ (STO) and $\mathrm{LaAlO}_{3}$ (LAO) [1] is an example of novel interface functionality with great potential for applications [2]. However, in spite of intense research efforts, the microscopic mechanism underlying such fascinating behavior is still controversial. A viable route to improve our understanding of STO/LAO interfaces is to investigate the properties of novel heterostructures realized with alternative overlayer materials. Alternative materials should be searched within wide band-gap insulating $\mathrm{ABO}_{3}$ perovskites showing a high quality growth on STO and having trivalent cations both on the $\mathrm{A}$ and the $\mathrm{B}$ site. On the basis of such considerations, $\mathrm{LaGaO}_{3}$ (LGO), a polar, wide band gap, pseudocubic perovskite, was selected and highly conducting interfaces were obtained in the LGO/STO system [3,4]. Although LAO/STO and LGO/STO show qualitatively similar transport properties, LGO/STO shows a slightly larger conductivity than LAO/STO (see Fig. 1a), independent of the oxygen pressure during growth. Here we use aberration corrected Z-contrast scanning transmission electron microscopy (Z-STEM) and atomically resolved electron energy loss spectroscopy (EELS) to gain insights into the similarities and differences between these two interface systems and their implications on the mechanism for electrical conductivity.

Our investigation has shown that both interfaces are atomically flat and coherent. An analysis of the relative lattice parameters of film and substrate was conducted by acquiring the position of the center of intensity for the atomic columns forming the A sublattice in HAADF images using our own Gatan Digital Micrograph script. The distances between adjacent columns were then averaged in the direction parallel to the interface to yield in-plane and out-of-plane lattice spacing. Both images and diffraction patterns confirmed that LGO and LAO films were single-domain and tetragonally strained with in-plane lattice constant equal to that of STO. We observed a peculiar difference between the two interfaces. As shown in Fig. 1b, while the $c$ spacing in the LAO/STO system is expanded by about $4 \%$ at the interface similar to prior literature reports $[5,6]$, an interfacial lattice distortion was not detected in the LGO/STO system. Orbital reconstruction and lattice distortions at the interface between LAO and STO are expected in theory and have been observed experimentally by linear dichroism in x-ray absorption spectroscopy (LD-XAS) [7] and optical second harmonic generation (SHG) [8]. Our Z-STEM investigations indicate that atomic distortions are less prominent in $\mathrm{LGO} / \mathrm{STO}$ than in LAO/STO. These results are in agreement with preliminary LD-XAS and SHG analyses on the same interfaces. Lattice polarization might arise to mitigate the dipole generated by the alternating charge within the film planes, considered as the origin of the polar catastrophe. As the LGO and the LAO systems show different degrees of interfacial lattice distortion, we expect a 
different overall polar layer response for the two systems and possible differences in either induced charge carriers or the threshold for conductivity.

To gain insights into the electronic state of interfacial Ti ions, we have analyzed the $\mathrm{O}-\mathrm{K}$ and $\mathrm{Ti}-\mathrm{L}_{3,2}$ edges in EEL spectra across the interfaces of both LAO/STO and LGO/STO. Our experimental results and EEL scattering simulations show that both interfaces can be considered atomically abrupt with occasional surface amorphous Ti species resulting from ion milling (see profiles in Figures 1c and 1d). Moreover, in both cases the valence of the interfacial Ti ions is reduced to an intermediate value between $4+$ and 3+. However, the fraction of interfacial Ti3+ is larger in LGO/STO than in LAO/STO, possibly indicating a larger charge transfer in the LGO system, which would be consistent with the lower resistivity measured in LGO/STO. The larger Ti3 + fraction might be related to the smaller atomic displacement observed in the LGO/STO system. A smaller ionic polarization at the LGO/STO interface would less efficiently counteract the electric dipole associated with the polar nature of the film and a larger charge reconstruction would be needed to avoid the polar catastrophe as compared to the LAO/STO case [9].
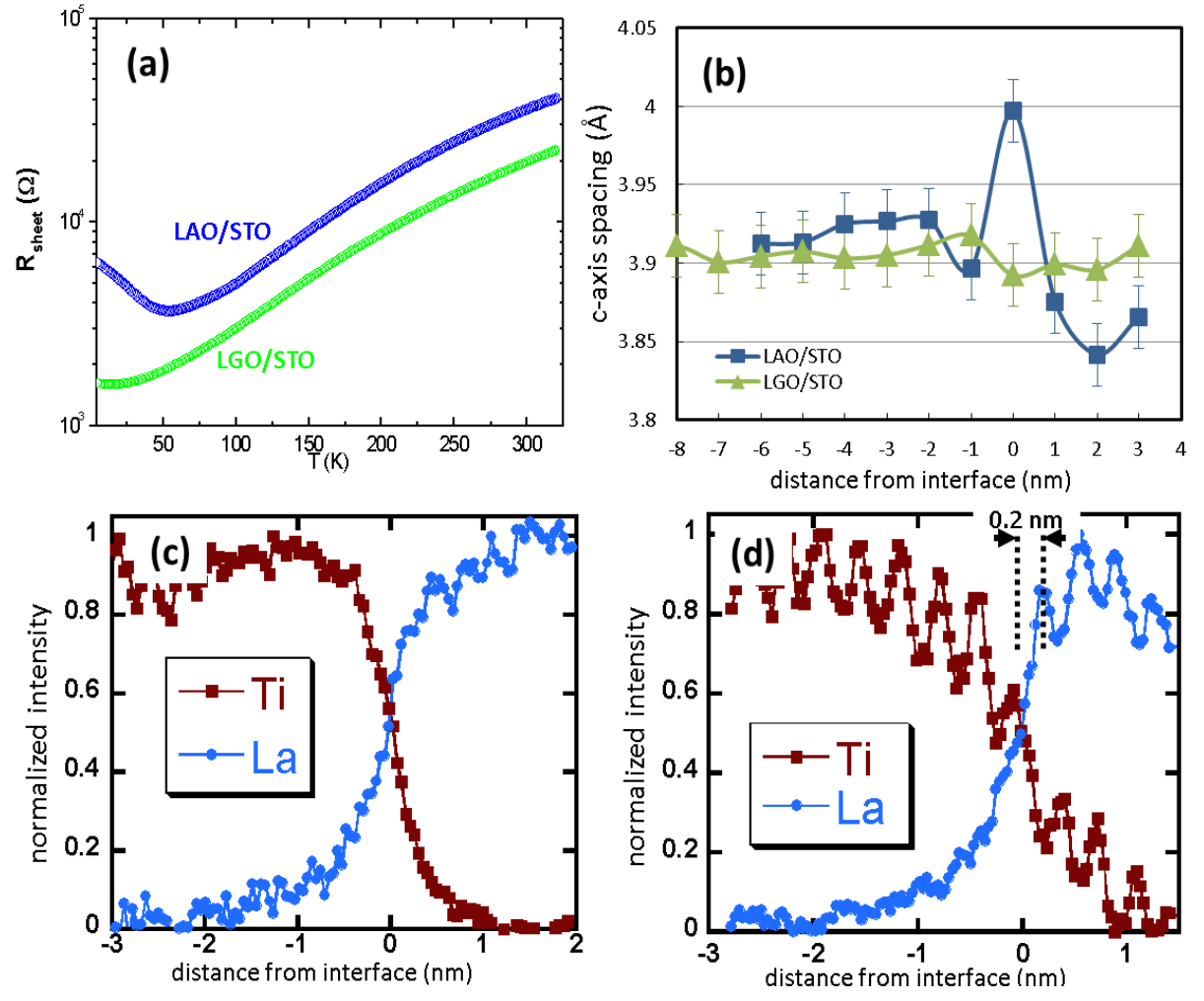

Fig. 1. Comparison between $\mathrm{LAO} / \mathrm{STO}$ and LGO/STO. (a) Sheet resistance vs temperature of 12 u.c. LAO and LGO interfaces grown at $\mathrm{P}\left(\mathrm{O}_{2}\right)=10^{-4}$ mbar. (b) c-axis spacing across LAO and LGO interfaces. (c) EELS integrated intensity profiles across LAO/STO. (d) EELS integrated intensity profiles across LAO/STO.

Figures (a), (c), and (d) adapted from [3]. Figure (b) adapted from C. Cantoni et al., in preparation.

[1]A. Ohtomo and H. Y. Hwang, Nature 427, (2004) 423.

[2] C. Cen et al., Science 323, (2009) 1026.

[3] P. Perna et al., Appl. Phys. Lett. 97, (2010) 152111.

[4] C. Aruta et al., Appl. Phys. Lett. 97, (2010) 252105.

[5] J.-L. Maurice et al., Phys. Stat. Sol. (a) 203, (2006) 2209-2214.

[6] V. Vonk et al., Phys. Rev. B 75, (2007) 235417.

[7] M. Salluzzo et al., Phys. Rev. Lett. 102, (2009) 166804.

[8] A. Rubano et al., Phys. Rev. B, in press

[9] Research sponsored by US DOE Office of Science, Division of Materials Science and Engineering. 